\title{
High-LET Radiotherapy
}

National Cancer Institute

\section{Source}

National Cancer Institute. High-LET Radiotherapy. NCI Thesaurus. Code C15849.

Radiotherapy using radioactive particles that deposit a high amount of energy by radiation per unit length of travel. 